\title{
Choroidal tuberculoma as a presenting sign of tuberculosis
}

This article was published in the following Dove Press journal:

International Medical Case Reports Journal

II November 2016

Number of times this article has been viewed

\author{
Nicolas Arej ${ }^{1,2}$ \\ Ali Fadlallah ${ }^{1,2}$ \\ Elias Chelala ${ }^{1,3}$ \\ 'Department of Ophthalmology, \\ Faculty of Medicine, Saint-Joseph \\ University, Beirut, ${ }^{2}$ Department of \\ Ophthalmology, Eye and Ear Hospital \\ International, Dbayeh, ${ }^{3}$ Department of \\ Ophthalmology, Hôtel-Dieu de France \\ Hospital, Beirut, Lebanon
}

\begin{abstract}
Choroidal tuberculoma is a rare ocular form of tuberculosis (TB) that raises both a diagnostic and a therapeutical challenge, especially when occurring without other manifestations of the disease. This study reports the case of a 27-year-old woman who had a unilateral drop of vision (20/100) with ocular pain. Her fundus examination revealed an elevated juxtapapillary choroidal mass measuring $892 \mu \mathrm{m}$ in diameter, as calculated by optical coherence tomography (OCT), and associated with a serous retinal detachment involving the macula. The diagnosis of choroidal tuberculoma was established by positive QuantiFERON-TB and tuberculin skin test. Laboratory and imaging workup ruled out pulmonary and systemic TB as well as other possible etiologies. Antituberculosis therapy was started and led to an improved visual acuity (20/30) and a shrinkage of the tuberculoma to a diameter of $499 \mu \mathrm{m}$ at 3 months. This is one of the few reported cases of solitary choroidal tuberculoma in a patient with no other sign of TB. It sheds light on the place of OCT in the diagnosis and follow-up of the choroidal mass, in terms of measuring the size of the mass and revealing the associated serous retinal detachment and the distinctive "contact sign" between the neurosensory retina and the retinal pigment epithelium (RPE)-choriocapillaris layer surmounting the tuberculoma.
\end{abstract}

Keywords: Mycobacterium tuberculosis, tuberculoma, choroid, choroidal mass, optical coherence tomography, OCT, QuantiFERON-TB

\section{Case report}

A 27-year-old woman was seen in the eye emergency department with a 10-day history of decreased vision and pain in her right eye. The visual acuity measured by the Snellen chart was limited to 20/100 in her right eye. The visual acuity in her left eye was 20/20. Fundus examination showed an elevated nonpigmented mass in the right eye that measured $\sim 2.5 \times 2.5$ disc diameters just superior and temporal to the optic disc (green arrows, Figure 1). There was a flat retinal detachment in the macula area, and optical coherence tomography (OCT) showed a choroidal elevated mass $(892 \mu \mathrm{m}$ diameter) associated with a serous retinal detachment (Figure 1). Differential diagnosis included choroidal neoplasia (primary tumor or metastasis), as well as infectious and inflammatory diseases. Malignancy was less likely considering the age of the patient and the presence of ocular pain, however could not be ruled out in the absence of another confirmed etiology. Blood workup showed a negative angiotensin-converting enzyme titer and negative toxoplasma and HIV serologies. The patient was considered to be at a high risk of contracting tuberculosis (TB) and had an unclear history of bacilli Calmette-Guérin vaccination (BCG) vaccination; therefore, she underwent both a QuantiFERON-TB test and a purified protein derivative skin testing. The former
Correspondence: Ali Fadlallah Department of Ophthalmology, Eye and Ear Hospital International, Dbayeh, P.O.Box 70-933 Naccache, Lebanon Tel +96I 4521130 Fax +961 4521139 Email fadlallahmd@gmail.com 


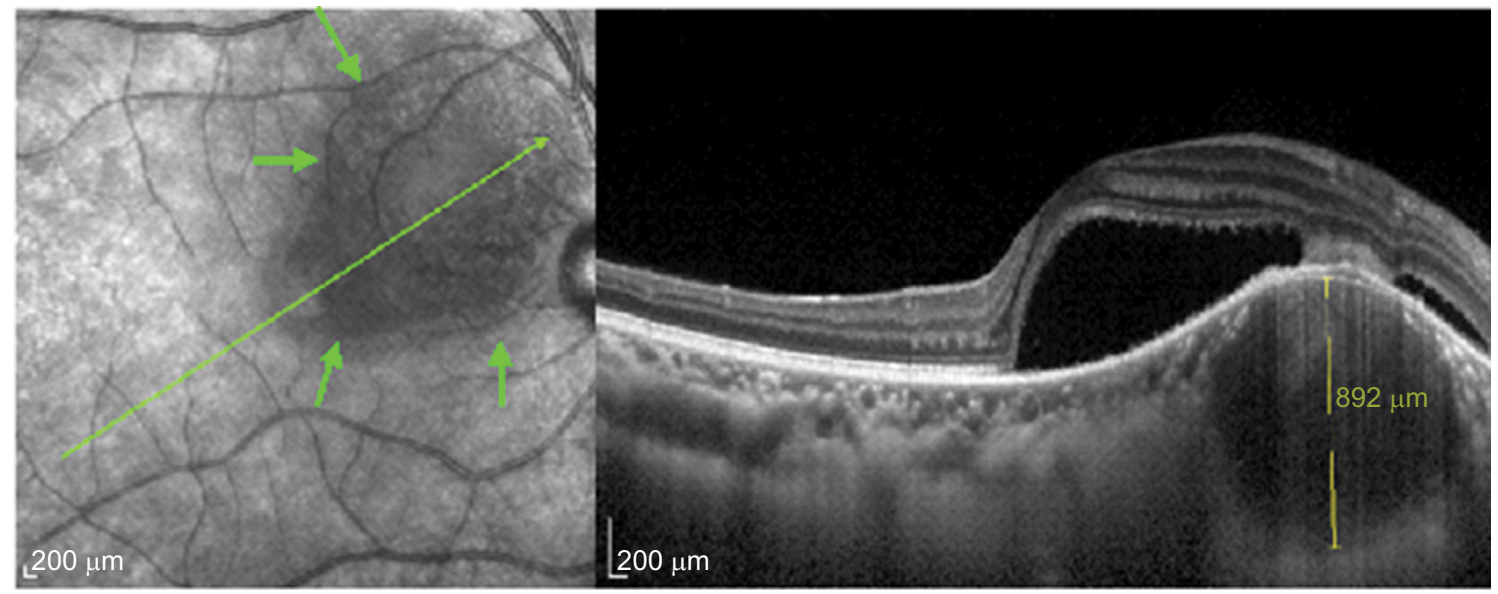

Figure I Chroidal mass associated with a serous retinal detachment. Note the contact zone between the retinal pigment epithelium-choriocapillaris layer and the neurosensory retina over the mass.

Note: The green arrows indicate the borders of the lesion as seen by OCT fundus photography.

Abbreviation: OCT, optical coherence tomography.

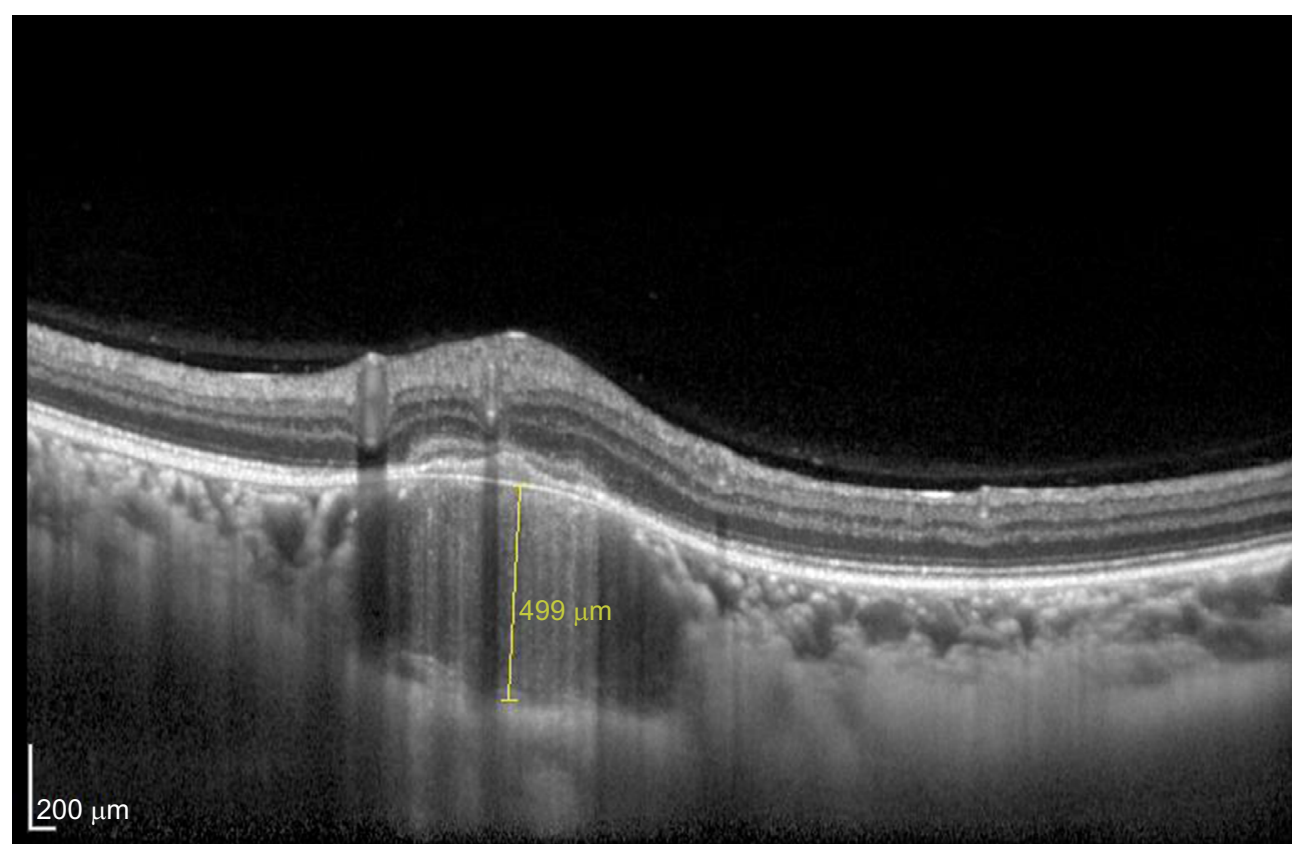

Figure 2 Shrinkage of the choroidal tuberculoma after 3 months of treatment.

was positive and the latter revealed an induration of $13 \mathrm{~mm}$. However, chest X-ray (CXR) was normal. The patient was then addressed to the internal medicine department for further investigations, systemic assessment, and treatment. No pulmonary or systemic involvement was detected. The diagnosis of isolated choroidal tuberculoma was retained and antituberculosis therapy (ATT) was started with daily doses of isoniazid $5 \mathrm{mg} / \mathrm{kg}$, rifampin $10 \mathrm{mg} / \mathrm{kg}$, and pyrazinamide 30 $\mathrm{mg} / \mathrm{kg}$, in addition to levofloxacin $750 \mathrm{mg} /$ day as a secondline molecule replacing ethambutol that we wanted to avoid due to its potential of ocular toxicity. This intensive phase lasted 2 months and was followed by a continuation phase of isoniazid and rifampin for at least 4 other months, depending on the response. Visual acuity in the right eye was 20/30 after 3 months of treatment, with a shrinked choroidal tuberculoma (499 $\mu \mathrm{m}$ in diameter) as shown by OCT (Figure 2).

\section{Discussion}

TB is an infection caused by Mycobacterium tuberculosis, commonly described as a systemic disease that involves not only the lungs but also many other organs, including the eye. ${ }^{1}$ Although ocular TB is a rare event ( $1 \%$ of all cases of TB), it can occur with or without evidence of systemic TB and can involve any part of the eye. ${ }^{2}$ The most common clinical presentation appears to be posterior uveitis, particularly choroidal TB. Multiple choroidal tubercles are reported to 
be the most common intraocular manifestation of tubercular posterior uveitis. Less commonly, intraocular TB may present as a large tuberculoma: a solitary yellowish or grayish white large lesion, generally located in the posterior pole. ${ }^{3}$ Rapid multiplication of the bacilli within a tuberculoma can cause tissue destruction through liquefactive necrosis, thus forming a surrounding exudative retinal detachment.

Rarely, a tuberculoma may be the presenting sign in a patient with no evidence of systemic disease. To our knowledge, only seven other cases of a choroidal tuberculoma without systemic evidence of TB and normal CXR findings have been reported. Tuberculin skin test (TST) was positive in five cases. ${ }^{3-6}$ In one case, the diagnosis was confirmed after histopathological examination of the enucleated eye. In another case, $M$. tuberculosis DNA amplification by polymerase chain reaction on aqueous humor sample confirmed the diagnosis. ${ }^{7}$ However, this technique lacks sensitivity, probably because of the thick cell wall of $M$. tuberculosis and its low bacterial load in the ocular fluids. ${ }^{1}$ In recent years, measurement of interferon gamma by QuantiFERON-TB or enzyme-linked immunosorbent spot has become a new technique to diagnose TB infection. QuantiFERON-TB is an indirect test for $M$. tuberculosis infection and has higher specificity than TST, as it is unaffected by previous BCG. ${ }^{8}$

Imaging techniques, such as fluorescein angiography and B-scan, can assist in excluding other diagnoses, especially intraocular tumors (e.g., melanoma) or infective abscesses. ${ }^{2}$ They were not performed in this case since the diagnosis was confirmed by laboratory tests and specific OCT findings. In fact, OCT scans through areas of suspected granuloma can be very helpful in differentiating choroidal granulomas from other noninflammatory conditions. Salman et al were the first to describe a distinctive feature of attachment between the retinal pigment epithelial-choriocapillaris layer and the neurosensory retina over the granuloma ("contact" sign). ${ }^{9}$ This was associated with surrounding subretinal fluid and inflammatory infiltrate in the deeper retinal layers. The "contact" sign and the subretinal fluid are clearly visible in Figure 1.

Visual recovery and choroidal tuberculoma involution to a flat inactive scar can occur with proper and rapid diagnosis and treatment. Systemic treatment with the first-line combination regimen comprising isoniazid, rifampin, pyrazinamide, and ethambutol for a total of 6-12 months has been accepted as standard therapy. If there is evidence of a clinical response to treatment after the 2-month initiation phase, a 4-month consolidation phase should follow using two first-line drugs (isoniazid and rifampin). Although symptoms may not completely resolve, especially in advanced disease, a demonstrable reduction in inflammation is expected..$^{10}$ In the present case, a favorable response to treatment was demonstrated by the shrinking of the tuberculoma on OCT at 3 months.

This was one of the rare cases of choroidal tuberculoma being the presenting sign of TB. Ophthalmologists should be aware that solitary choroidal tuberculoma can occur in immunocompetent individuals. It is believed that OCT is an easy and practical method for not only suspecting the diagnosis but also monitoring disease evolution and response to treatment.

\section{Acknowledgment}

The patient provided written informed consent for publication of this paper and accompanying images.

\section{Disclosure}

The authors report no conflicts of interest in this work.

\section{References}

1. Yeh S, Sen HN, Colyer M, Zapor M, Wroblewski K. Update on ocular tuberculosis. Curr Opin Ophthalmol. 2012;23(6):551-556.

2. Bodaghi B, LeHoang P. Ocular tuberculosis. Curr Opin Ophthalmol. 2000;11(6):443-448.

3. Mansour AM, Haymond R. Choroidal tuberculomas without evidence of extraocular tuberculosis. Graefes Arch Clin Exp Ophthalmol. 1990;228(4):382-383.

4. Berinstein DM, Gentile RC, McCormick SA, Walsh JB. Primary choroidal tuberculoma. Arch Ophthalmol Chic Ill 1960. 1997;115(3):430-431.

5. Levecq LJ, De Potter P. Solitary choroidal tuberculoma in an immunocompetent patient. Arch Ophthalmol Chic Ill 1960. 2005;123(6): 864-866.

6. Alaraj AM, Al-Dhibi H, Al-Mezaine HS, Abu El-Asrar AM. Solitary presumed choroidal tuberculomas masquerading as choroidal tumors. Saudi Med J. 2013;34(1):86-90.

7. Sarvananthan N, Wiselka M, Bibby K. Intraocular tuberculosis without detectable systemic infection. Arch Ophthalmol Chic Ill 1960 1998;116(10):1386-1388

8. Kang YA, Lee HW, Yoon HI, et al. Discrepancy between the tuberculin skin test and the whole-blood interferon gamma assay for the diagnosis of latent tuberculosis infection in an intermediate tuberculosis-burden country. JAMA. 2005;293(22):2756-2761.

9. Salman A, Parmar P, Rajamohan M, Vanila CG, Thomas PA, Jesudasan CAN. Optical coherence tomography in choroidal tuberculosis. Am J Ophthalmol. 2006;142(1):170-172.

10. Zhang M, Zhang J, Liu Y. Clinical presentations and therapeutic effect of presumed choroidal tuberculosis. Retina Phila Pa. 2012;32(4):805-813. 
The International Medical Case Reports Journal is an international, peer-reviewed open-access journal publishing original case reports from all medical specialties. Previously unpublished medical posters are also accepted relating to any area of clinical or preclinical science. Submissions should not normally exceed 2,000 words or
4 published pages including figures, diagrams and references. The manuscript management system is completely online and includes a very quick and fair peer-review system, which is all easy to use. Visit http://www.dovepress.com/testimonials.php to read real quotes from published authors.

Submit your manuscript here: https://www.dovepress.com/international-medical-case-reports-journal-journal 